\title{
Xiaochaihu decorction relieves liver fibrosis caused by Schistosoma japonicum infection via the HSP47/TGF- $\beta$ pathway
}

\author{
Yuzheng Huang ${ }^{1,2,3^{*}}$, Jin Lu ${ }^{1,2,3}$, Yongliang $X u^{1,2,3}$, Chunrong Xiong ${ }^{1,2,3}$, Deshen Tong ${ }^{1,2,3}$, Nannan Hu $u^{1,2,3}$ \\ and Haitao Yang ${ }^{1,2,3^{*}}$
}

\begin{abstract}
Background: Hepatic fibrosis caused by chronic infection with Schistosoma japonica remains a serious public health problem in the world. Symptoms include inflammation, liver granuloma and fibrosis, whilst treatment options are still limited. This study aims to investigate whether and how traditional Chinese medicine Xiaochaihu decoction (XCH) could mitigate liver fibrosis caused by S. japonicum infection.
\end{abstract}

Methods: BALB/c mice were infected with S. japonicum cercariae and treated with XCH for 16 weeks. Liver pathological changes were assessed by H\&E and Masson staining. NIH3T3 and Raw264.7 cells were treated with S. japonicum egg antigens with or without XCH treatment. Quantitative real-time PCR, western blot, immunfluorescence and ELISA were performed to determine the changes of levels of fibrogenic markers.

Results: XCH protected mouse liver from injuries and fibrosis caused by S. japonicum infection and considerably reduced egg burden in a dose-dependent manner. Infection with S. japonicum caused elevation of serum ALT, AST, ALP, HA and PIIINP levels and reduction of ALB and GLOB levels, which was markedly suppressed by XCH. The upregulation of TGF- $\beta 1$, Hsp47, a-SMA, Col1A1 and Col3A1 in S. japonicum-infected mouse liver was also significantly inhibited by XCH. Schistosoma japonicum egg antigens promoted the expression of Hsp47, TGF- $\beta 1$, Timp-1, a-SMA, Col1A1 and Col3A1 in NIH3T3 cells, and TGF- $\beta 1$, CTGF, LL-13, IL-17 and IL-6 in Raw264.7 cells, which was inhibited by XCH, LY2157299 and shRNA-Hsp47.

Conclusions: These results demonstrated that the hepatic protective effects of Xiaochaihu decoction were mediated by HSP47/TGF- $\beta$ axis.

Keywords: Xiaochaihu decorction, Schistosoma japonicum, Hepatic fibrosis, HSP47, TGF- $\beta$

\section{Background}

Schistosomiasis is a serious parasitic disease and caused by six species of parasites belonging to the genus Schistosoma, which are uniquely geographically distributed in the tropical and subtropical areas worldwide [1].

\footnotetext{
*Correspondence: yzhyz01@163.com; yht@jscdc.cn

${ }^{1}$ National Health Commission Key Laboratory of Parasitic Disease Control and Prevention, Jiangsu Provincial Key Laboratory on Parasite and Vector Control Technology, Jiangsu Institute of Parasitic Diseases, 117 Meiyuan Yangxiang, Wuxi 214064, Jiangsu, China

Full list of author information is available at the end of the article
}

Schistosomiasis is highly debilitating with three distinct phases of clinical disease progression: acute infection; established active infection; and late chronic infection $[2,3]$. Chronic and advanced schistosomiasis remain a serious public health problem in China. The marshland areas with infected snails in Anhui, Jiangxi, Hunan, and Hubei provinces are the major endemic region [4]. Schistosoma japonicum eggs cause granulomatous inflammation in the host liver during the acute phase and lead to chronic liver damage, which might progress 
to the hepatosplenic schistosomiasis with egg granuloma deposition and fibrosis on the vascular wall [5-7].

Liver cirrhosis is the advanced stage of fibrosis due to chronic inflammation [8]. Hepatic fibrosis could be induced by infectious agents such as the bacterium Helicobacter hepaticus [9] and the parasite Schistosoma [10]. Hepatic Kupffer cells are activated and bone marrow derived macrophages recruited to the liver upon exposure to inflammatory inducers or liver injuries [11]. Macrophage-produced TGF- $\beta 1$ activates otherwise quiescent hepatic stellate cells which in turn secrete an extracellular matrix, leading to fibrosis $[8,11]$.

Heat-shock protein 47 (HSP47) is an ER-resident molecular chaperone that binds specifically to procollagen [12]. The expression pattern of HSP47 closely correlates with collagen expression [13]. Mice with whole body Hsp47 knockout (KO) die after 11.5 days post-coitus (dpc) due to almost complete loss of the mature type I collagen and fibril structures of type I collagen in embryonic mesenchymal tissues [14]. Chondrocyte-specific Hsp47 KO mice die around birth with severe generalized chondrodysplasia and bony deformities due to reduced levels of type II and type XI collagen [15]. HSP47 has been shown to regulate the biosynthesis, processing, transport, secretion and assembly of collagens $[16,17]$. Thus, Hsp47 is used as a target for treating collagen-related diseases including skin and lung fibrosis $[18,19]$.

Xiaochaihu decorction $(\mathrm{XCH})$ was first described in the Shang Han Lun to treat febrile diseases by the physician Zhong-Jing Zhang around AD200, including Radix Bupleuri (Chinese thorowax root), Radix Scutellariae (huangqin or baical skullcap root), Rhizoma Pinelliae (banxia or pinellia tuber), Radix Ginseng (renshen or ginseng), Radix Glycyrrhizae (gancao or licorice root), Rhizoma Zingiberis Recens (shengjiang or fresh ginger) and Fructus Jujubae (dazhao or Chinese date) [20]. $\mathrm{XCH}$ has been shown to protect against experimental liver injuries [21, 22], prevent and treat experimental hepatic and pancreatic fibrosis [23-25]. This study aims to investigate the effects of $\mathrm{XCH}$ on hepatic fibrosis of $S$. japonicum infected mice and the underlying molecular mechanism.

\section{Methods}

\section{Cell culture and treatment}

NIH 3T3 cells and Raw264.7 cells were obtained from the Cell Bank of Shanghai Institute of Biochemistry and Cell Biology, Chinese Academy of Sciences (Shanghai, China). The cells were maintained in DMEM medium containing $10 \% \mathrm{FBS}$ and incubated at $37^{\circ} \mathrm{C}$ with $5 \%$ humidified $\mathrm{CO}_{2}$. NIH3T3 were treated with XCH, LY2157299, TGF- $\beta 1$ and shRNA-HSP47 for $48 \mathrm{~h}$. Raw264.7 cells were treated with XCH and LY2157299 for $48 \mathrm{~h}$.
Schistosoma japonicum egg antigen $(0.01 \mathrm{~g} / \mathrm{ml}$ in phosphate-buffered saline) was obtained from the Jiangsu Institute of Parasitic Diseases (Wuxi, China) and diluted to the working concentration $(10 \mu \mathrm{g} / \mathrm{ml})$ in DMEM containing $2 \%$ FBS immediately before use.

\section{Western blotting}

The western blotting procedure was performed as described in our previous reports [26]. Briefly, after required treatments, the total protein of the cell samples was isolated using radioimmunoprecipitation assay (RIPA) lysis buffer. Equal amounts of total protein were separated by appropriate SDS-PAGE and transferred to a polyvinylidene fluoride (PVDF) membrane. After blocking with skimmed milk, the PVDF membrane was incubated with specific primary antibodies followed by incubation with the corresponding secondary antibodies. Protein bands were detected using the Bio-Rad ChemiDocTM (Hercules, CA, USA). $\beta$-actin was used as an internal control.

\section{Real-time quantitative polymerase chain reaction (RT-qPCR) analysis}

NIH3T3 were treated with XCH, LY2157299, TGF- $\beta 1$ and shRNA-HSP47. Raw 264.7 cells were treated with $\mathrm{XCH}$ and LY2157299. Total RNA was subsequently extracted from cells using RNAiso plus (Takara, Dalian, China) according to the manufacturer's instructions. The total RNA was reverse-transcribed into cDNA using an M-MLV Reverse Transcriptase kit (Invitrogen, Shanghai, China), and the resultant cDNA mixture was diluted 10-fold in RNase-free $\mathrm{ddH}_{2} \mathrm{O}$. RT-qPCR amplification was performed using SYBR Premix Ex Taq ${ }^{\mathrm{TM}}$ kit (Takara) in a $20 \mu \mathrm{l}$ reaction containing $0.4 \mu \mathrm{l}$ of each primer, $0.4 \mu \mathrm{l}$ SYBR Green Dye and $2 \mu \mathrm{l}$ of cDNA. The PCR primers are listed in Additional file 1: Table S1. RT-qPCR was carried out on a Roche LightCycler 480II (Roche Diagnostics, Shanghai, China) using the following program: $95^{\circ} \mathrm{C}$ for $30 \mathrm{~s}$; followed by 40 cycles of 95 ${ }^{\circ} \mathrm{C}$ for $5 \mathrm{~s}$ and $58{ }^{\circ} \mathrm{C}$ for $34 \mathrm{~s}$. The relative gene expression level was calculated with $2^{-\Delta \Delta C q}$ method using $\beta$-actin as an internal control.

\section{Immunofluorescence staining}

The localization of COL3Al and COL1Al were analyzed by immunofluorescence staining. Cells were fixed with $4 \%$ formaldehyde in PBS containing $1 \%$ sucrose at $37^{\circ} \mathrm{C}$ for $15 \mathrm{~min}$. The fixative was then removed and the samples were permeabilized with $0.1 \%$ of Triton$\mathrm{X} 100$ at $4{ }^{\circ} \mathrm{C}$ for $10 \mathrm{~min}$. This was followed by a blocking step with $1 \%$ bovine serum albumin (BSA)/PBS at $37^{\circ} \mathrm{C}$ for $5 \mathrm{~min}$. After blocking, the cells were incubated with anti-COL3Al (Cat\# ab7778, 1:200 dilution; Abcam, Cambridge, MA, USA) or COL1Al (Cat\# ab34710, 1:500 
dilution; Abcam) in $1 \%$ BSA/PBS for $1 \mathrm{~h}$ at $37^{\circ} \mathrm{C}$. Cells were then incubated with a $\mathrm{Cy}-3$ conjugated secondary goat anti-rabbit antibody (Jackson ImmunoResearch, West Grove, PA, USA) for $30 \mathrm{~min}$ at $4{ }^{\circ} \mathrm{C}$. The samples were mounted in DAPI to stain the nuclei. Images of the stained substrates were taken via a Leica IX71 fluorescent microscope (Leica Microsystems GmbH, Wetzlar, Germany).

\section{ELISA assay}

Raw264.7 cells $\left(1 \times 10^{4}\right.$ cells/well $)$ were plated in 96-well plates for $24 \mathrm{~h}$ and then incubated with or without $S$. japonicum egg antigens for $48 \mathrm{~h}$. The culture media were collected for the CTGF, TGF- $\beta 1$, IL-13, IL-17 and IL-6 assays. The concentrations of CTGF, TGF- $\beta 1$, IL- 13 , IL-17 and IL-6 in the cell culture media were determined by commercial ELISA kits (RJ17277, RJ17947, RJ16937, RJ16942, RJ16958 respectively, Renjie Bio (http://www. rjkit.com/products.html), Shanghai, China) according to the manufacturer's instructions.

\section{Parasites and animals}

Cercariae of S. japonicum (Chinese strain) were obtained from infected Oncomelania hupensis snails fed at the Vector Biology Laboratory in our institute using a standard procedure [27]. Sixty healthy adult female BALB/c mice with body weights of $c .20 \mathrm{~g}$ were purchased from Yangzhou University.

Mice were randomly assigned into two groups, a control group and an infected group. Infection of mice with 15 S. japonicum cercariae was carried out through shaved abdominal skin for $30 \mathrm{~min}$ and no other treatment or manipulation of the animals was involved. Using the same procedure, the control mice were exposed to physiological saline. Then the uninfected mice were divided into two groups (10 mice in each group) to receive saline or Xiaochaihu decoction $(\mathrm{XCH}-\mathrm{H})$. The infected mice were divided into four groups (10 mice in each group) to receive saline or one of three doses $(\mathrm{XCH}-\mathrm{L}, \mathrm{XCH}-\mathrm{M}$ and $\mathrm{XCH}-\mathrm{H}$ ) of Xiaochaihu decoction by gavage, respectively.

\section{Xiaochaihu decoction}

$\mathrm{XCH}$ was made from $24 \mathrm{~g}$ bupleurum, $9 \mathrm{~g}$ astragalus, $9 \mathrm{~g}$ ginseng, $9 \mathrm{~g}$ pinellia, $9 \mathrm{~g}$ licorice, $9 \mathrm{~g}$ ginger root and $40 \mathrm{~g}$ jujube, which was boiled in $500 \mathrm{ml} \mathrm{H}_{2} \mathrm{O}$ and simmered until the volume reduced to $100 \mathrm{ml}$. The dosage of XCH per mouse was as follows: $\mathrm{XCH}-\mathrm{L}(5 \mathrm{ml} / \mathrm{kg} /$ day); $\mathrm{XCH}-\mathrm{M}$ $(15 \mathrm{ml} / \mathrm{kg} /$ day $)$; and XCH-L $(30 \mathrm{ml} / \mathrm{kg} /$ day $)$. The final concentration for cell culture was $1.67 \mu \mathrm{l} / \mathrm{ml}$.

\section{Identification of infected mice}

Stool samples were also collected every week after infection and examined using kato-katz thick smears [27].
Infected with S. japonicum mice were successfully identified with parasite eggs found in the feces of all mice at 6 weeks post-infection. The liver was extracted from all mice 16 weeks post-infection. Hematoxylin and eosin (H\&E) and Masson staining of liver tissue was performed to assess pathological changes.

\section{Liver function and fibrosis biomarkers}

Mouse serum alanine aminotransferase (AST), aspartate aminotransferase (ALT), and alkaline phosphatase (ALP) activities were measured using specific assay kits (ab105134 for AST, ab105135 for ALT, ab83369 for AP, Abcam, Shanghai, China). Serum hyaluronic acid (HA), $\mathrm{N}$-terminal propeptide of collagen III (PIIINP), albumin (ALB), and globulin (GLOB) levels were analyzed with commercial ELISA kits (CSB-E08121m for HA, CUSABIO, Houston, TX, USA; LS-F25207 for PIIINP, LSBio, Seattle, WA; ab108791 for albumin, and ab151276 for globulin, Abcam, Shanghai, China).

\section{Statistical analysis}

Results are expressed as the mean \pm standard deviation. Statistical analyses were performed with Graphpad Prism 6 (Graphpad, San Diego, CA, USA). The differences among treatment groups were analyzed by a one-way ANOVA followed by Tukey's post-hoc test. A P-value less than 0.05 was considered statistically significant.

\section{Results}

$\mathrm{XCH}$ alleviated liver granuloma and fibrosis in mice caused by S. japonicum

Schistosoma japonicum infection caused destruction of the histological structure of mouse liver with a large number of $S$. japonicum eggs present around the portal veins (Fig. 1a, b). Meanwhile, S. japonicum-infected mouse liver significantly increased fibrotic tissue compared to uninfected healthy mice (Fig. 2). Xiaochaihu decoction treatment markedly reduced the amount of $S$. japonicum eggs (ANOVA: $F_{(5,54)}=115.2, P<0.001$ ) (Fig. 1), the size of single liver granuloma and fibrotic tissue (ANOVA: $F_{(5,54)}=111.4, P<0.001$ ) (Fig. 2 ) in infected mouse livers in a dose-dependent manner.

\section{$\mathrm{XCH}$ improved hepatic dysfunction and fibrosis caused by S. japonicum}

Schistosoma japonicum infection resulted in a drastic increase in serum activities of alanine aminotransferase (ALT), aspartate aminotransferase (AST), alkaline phosphatase (ALP) as well as hyaluronic acid (HA) and $\mathrm{N}$-terminal propeptide of collagen III (PIIINP) levels and decreases of albumin (ALB) and globulin (GLOB) levels (Table 1). Xiaochaihu decoction significantly decreased ALT, AST and ALP activities and HA and PIIINP levels 


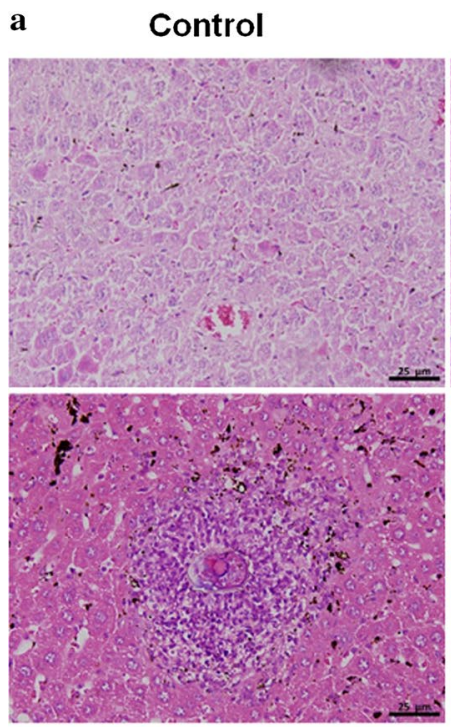

$\mathrm{M}+\mathrm{XCH}-\mathrm{L}$

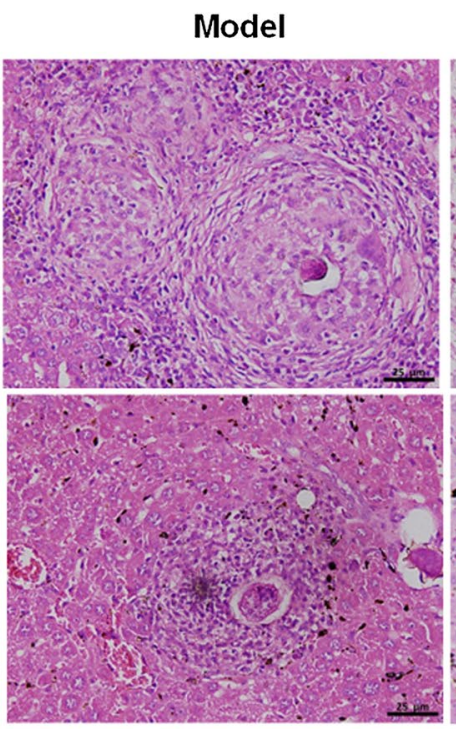

$\mathrm{M}+\mathrm{XCH}-\mathrm{M}$

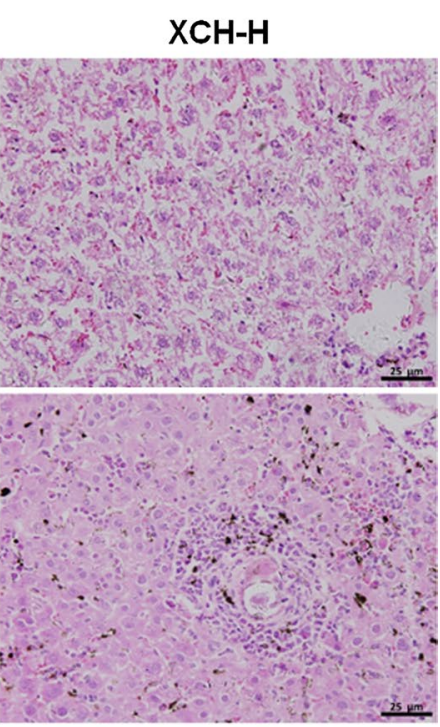

$\mathrm{M}+\mathrm{XCH}-\mathrm{H}$

b

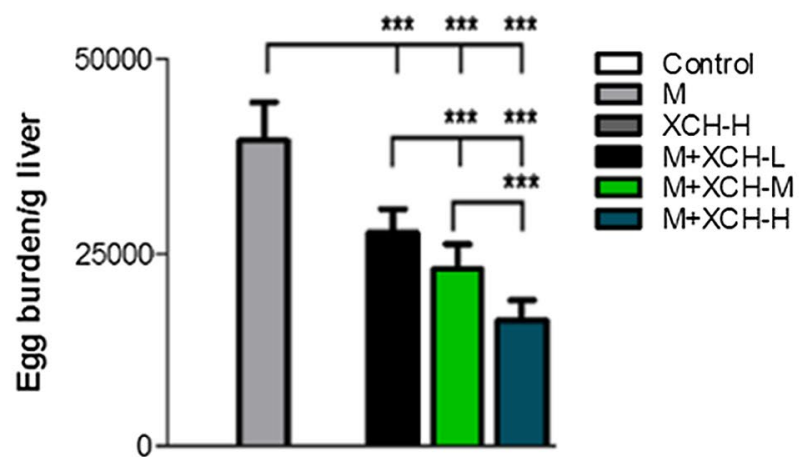

Fig. 1 Xiaochaihu $(X C H)$ decoction reduced Schistosoma japonicum burden of mouse liver. BALB/c mice were infected with 15 S. japonicum cercariae and treated with either saline or Xiaochaihu decoction. Mice were sacrificed and liver tissues were collected 16 weeks post-infection. a H\&E staining showing the pathological changes and S. japonicum eggs in mouse liver. b Statistical differences in egg burden of infected mice. Abbreviations: M, Model, S. japonicum infection; XCH-L (M, H), Xiaochaihu decoction low (middle, high) dose. Scale-bars: a, $25 \mu \mathrm{m}$. ${ }^{* * *} P<0.0001$

(decrease range of 22-57\%) while increased levels of ALB (51\%) and GLOB (27\%) were detected in mice infected with S. japonicum. (Table 1).

\section{$\mathrm{XCH}$ inhibited upregulation of hepatic fibrogenic genes caused by infection with S. japonicum}

As $\mathrm{XCH}$ relieved hepatic pathological changes caused by infection with $S$. japonicum including liver granuloma and fibrosis, we next assessed the expression levels of genes related to hepatic fibrosis. Schistosoma japonicum infection caused substantial upregulation of hepatic TGF- $\beta 1$, Hsp47, $\alpha$-SMA, Col1A1 and Col3A1 at both mRNA (Fig. 3a) and protein (Fig. 3b) levels. Xiaochaihu decoction dose-dependently inhibited the overexpression of these fibrotic genes in $S$. japonicum-infected mouse livers (ANOVA: $\alpha$-SMA, $F_{(5,54)}=67.3, P=0.0012$;
Col1A1, $F_{(5,54)}=252.2, P<0.0001 ; \mathrm{HSP} 47, F_{(5,54)}=73.3$, $P=0.0010$; COL3A1, $F_{(5,54)}=173.4, P=0.0002$; TGF$\beta 1, F_{(5,54)}=70.9, P=0.0011$ ) (Fig. 3).

\section{$\mathrm{XCH}$ inhibited fibroblast activation and collagen production through HSP47 and TGF- $\beta$}

To elucidate the mechanism governing the inhibition of XCH on S. japonicum infection caused hepatic fibrosis, we treated NIH3T3 mouse fibroblast with $S$. japonicum egg antigens in the presence or absence of TGF- $\beta$ or shRNA targeting Hsp47 (shRNA-Hsp47). Schistosoma japonicum egg antigens strongly stimulated the expression of Hsp47, TGF- $\beta 1$, Timp-1, $\alpha$-SMA, Col1A1 and Col3A1 in NIH3T3 cells, which was significantly inhibited by $\mathrm{XCH}$, TGF- $\beta$ receptor 


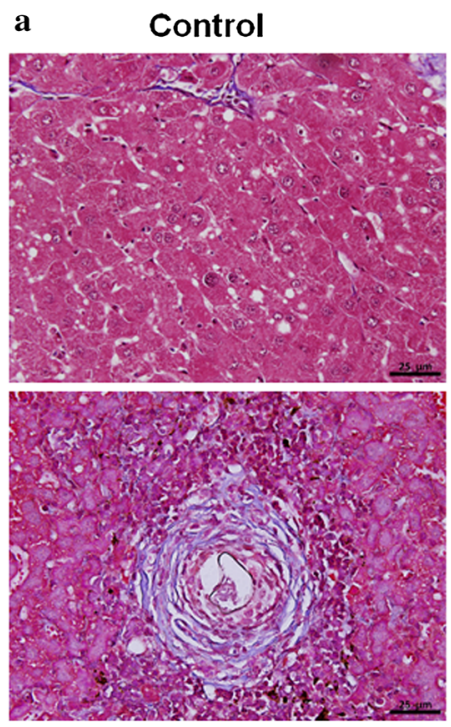

$\mathrm{M}+\mathrm{XCH}-\mathrm{L}$

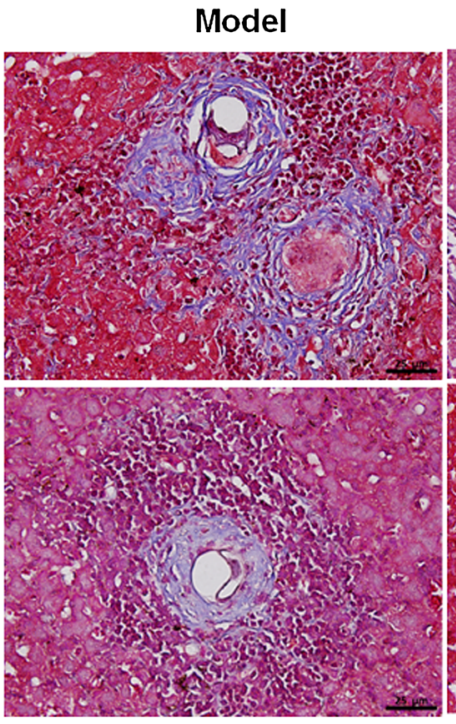

$\mathrm{M}+\mathrm{XCH}-\mathrm{M}$

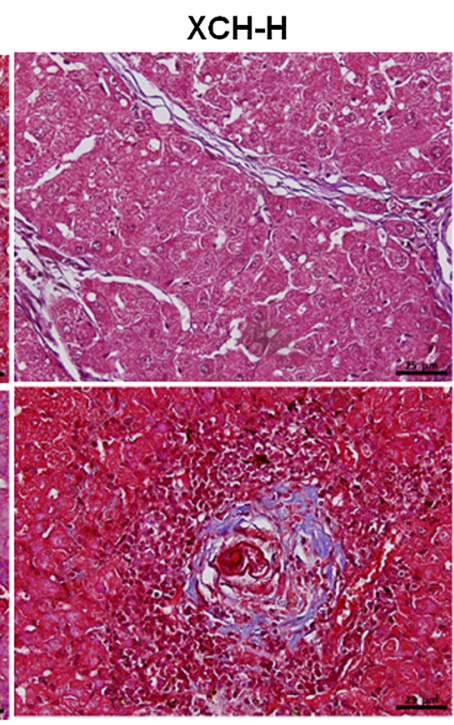

$\mathrm{M}+\mathrm{XCH}-\mathrm{H}$

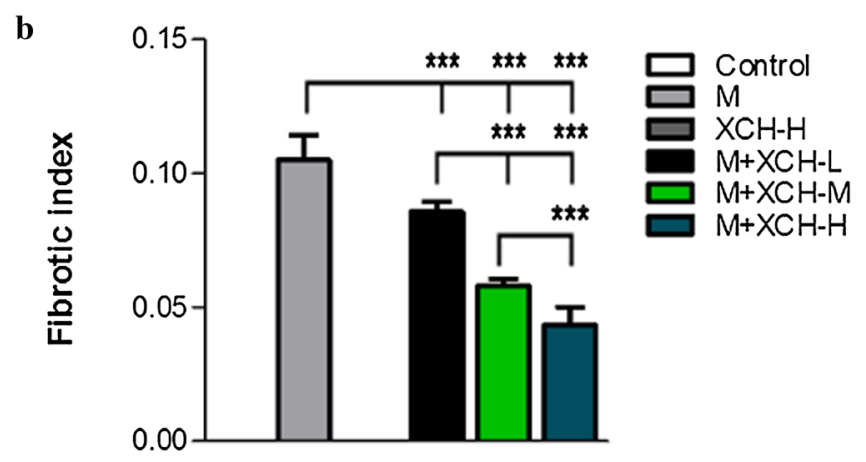

Fig. 2 Xiaochaihu $(X C H)$ decoction reduced Schistosoma japonicum caused liver fibrosis in mice. BALB/C mice were infected with 15 S. japonicum cercariae and treated with either saline or Xiaochaihu decoction. Mice were sacrificed and liver tissues were collected 16 weeks post-infection. a Masson staining showing the amount of fibrotic tissue in mouse liver. b Statistical differences in egg burden of infected mice. Abbreviations: $\mathrm{M}$, Model, S. japonicum infection; XCH-L (M, H), Xiaochaihu decoction low (middle, high) dose. Scale-bars: a, $25 \mu$ m. ${ }^{* * *} P<0.0001$

Table 1 Parameters of hepatic function and fibrosis

\begin{tabular}{|c|c|c|c|}
\hline & Control & SJ & $\mathrm{SJ}+\mathrm{XCH}$ \\
\hline ALT (U/I) & $22.7 \pm 4.7$ & $86.3 \pm 11.0^{*}$ & $40.7 \pm 7.8^{* \#}$ \\
\hline AST (U/I) & $116.7 \pm 15.3$ & $169.0 \pm 14.0^{*}$ & $132.0 \pm 15.9^{* \#}$ \\
\hline $\operatorname{ALP}(\mathrm{U} / \mathrm{I})$ & $31.3 \pm 0.6$ & $73.3 \pm 4.7^{*}$ & $42.7 \pm 3.1^{* \#}$ \\
\hline $\operatorname{ALB}(g / l)$ & $42.5 \pm 2.3$ & $23.3 \pm 2.8^{*}$ & $35.1 \pm 0.8^{* \#}$ \\
\hline GLOB (g/l) & $50.8 \pm 3.0$ & $33.5 \pm 4.2^{*}$ & $42.7 \pm 2.6^{* \#}$ \\
\hline ALB/GLOB & 0.8 & 0.7 & 0.8 \\
\hline $\mathrm{HA}(\mathrm{g} / \mathrm{l})$ & $411.7 \pm 31.8$ & $1924.3 \pm 179.8^{*}$ & $1168.7 \pm 155.9^{* \#}$ \\
\hline PIIINP (g/l) & $1.9 \pm 0.1$ & $3.7 \pm 0.3^{*}$ & $2.2 \pm 0.2^{\#}$ \\
\hline
\end{tabular}

Note: Data are shown as mean \pm SD

* $P<0.05$ compared to control, ${ }^{\#} P<0.05$ compared to $S J$

Abbreviations: SJ, Schistosoma japonicum; XCH, Xiaochaihu decoction; ALB, albumin; ALP, alkaline phosphatase, ALT, alanine aminotransferase; AST, aspartate aminotransferase; GLOB, globulin, HA, hyaluronic acid; PIIINP, $\mathrm{N}$-terminal propeptide of collagen III
1 (TGF- $\beta$ R1) inhibitor LY2157299 and shRNA-Hsp47 (ANOVA: $m R N A$ : a-SMA, $F_{(7,16)}=533.7, P<0.0001$; Col1A1, $F_{(7,16)}=525.2, P<0.0001 ; \operatorname{HSP} 47 ; F_{(7,16)}=$ 127.9, $P=0.0003$, COL3A1, $F_{(7,16)}=443.7, P<0.0001$; TGF- $\beta 1, F_{(7,16)}=1701.7, P<0.0001$; TIMP- $1, F_{(7,16)}$ $=86.9, P=0.0007$. Protein: a-SMA, $F_{(7,16)}=147.4, P$ $<0.0001$; Col I, $F_{(7,16)}=285.3, P<0.0001 ; \mathrm{HSP} 47, F_{(7}$, ${ }_{16)}=15528.6, P<0.0001$; COL III, $F_{(7,16)}=465.2, P<$ 0.0001; TIMP-1, $F_{(7,16)}=129.8, P=0.0003$ ) (Fig. 4a, b). Exogenous TGF- $\beta 1$ further augmented the upregulation of fibrotic genes by $S$. japonicum egg antigens, which was markedly suppressed by XCH (Fig. 4a, b). Moreover, the deposition of collagen 1 (Fig. 5a) and collagen 3 (Fig. 5b) was also drastically stimulated by $S$. japonicum egg antigens and further increased by TGF$\beta 1$, which was inhibited by $\mathrm{XCH}$ and shRNA-Hsp47. 


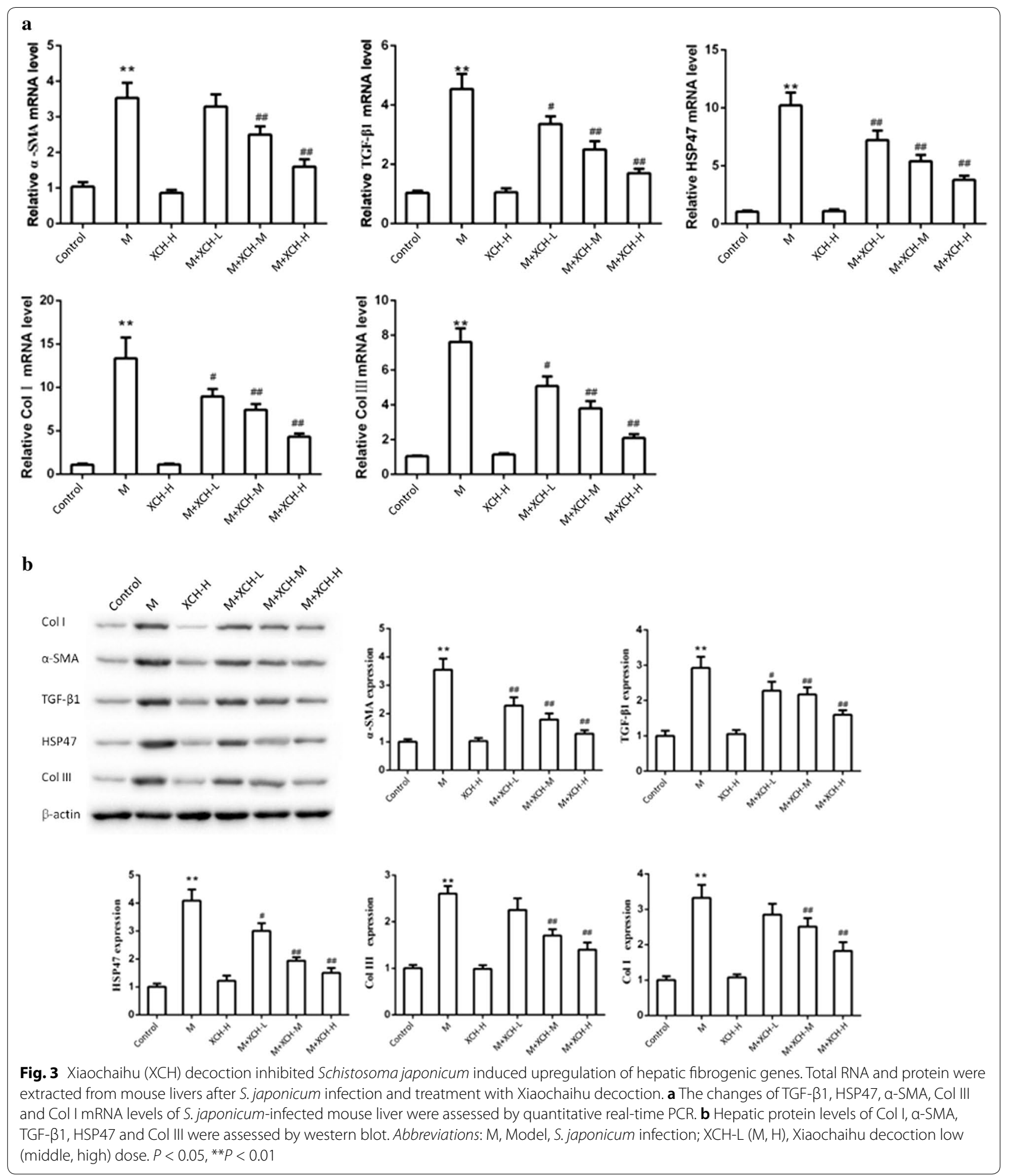




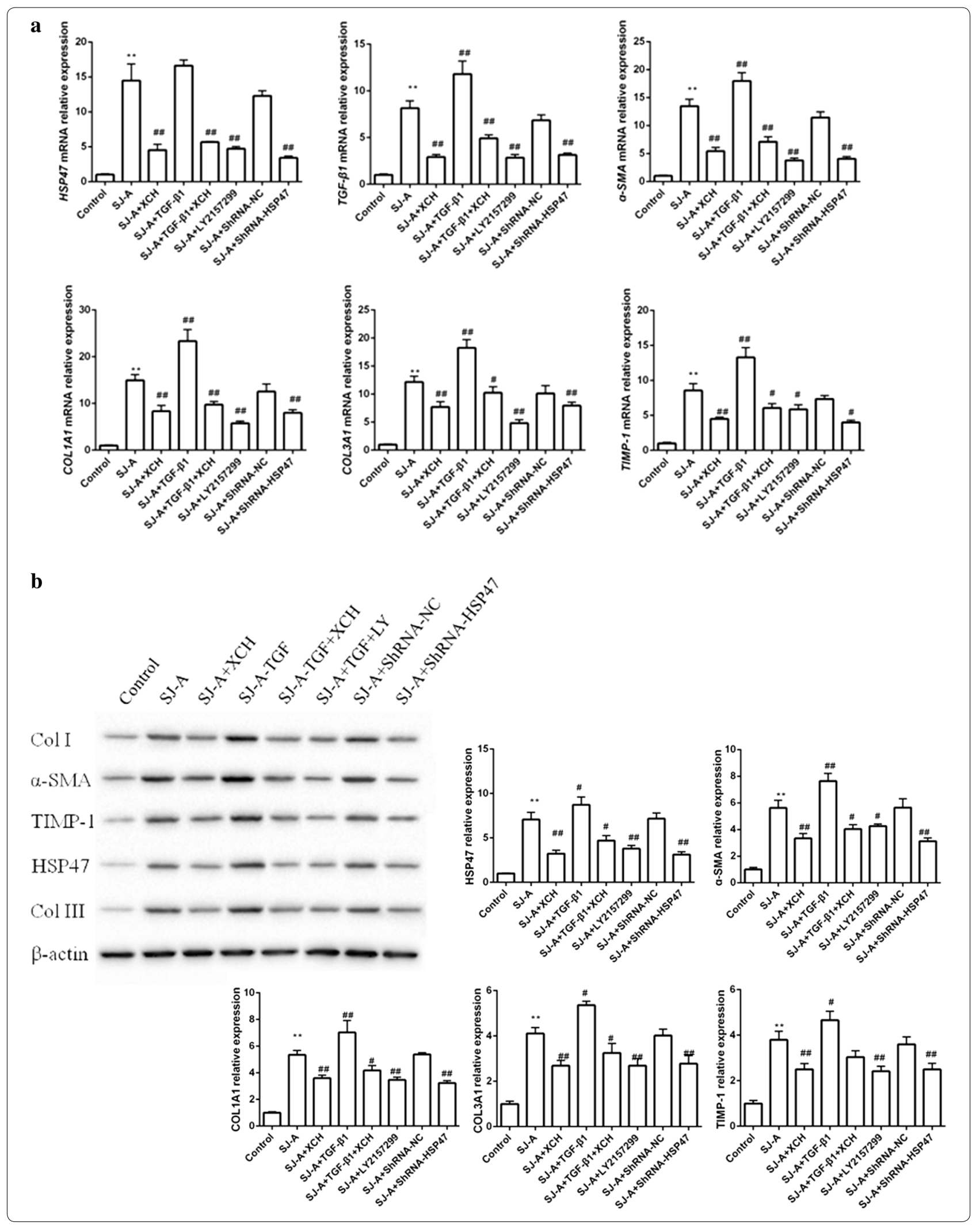


(See figure on previous page.)

Fig. 4 Xiaochaihu $(X C H)$ decoction inhibited Schistosoma japonicum induced fibroblast activation. a Quantitative real-time PCR showed that S. japonicum egg antigens induced upregulation of HSP47, TGF- $\beta 1$, Timp-1, a-SMA, Col1A1 and Col3A1 in NIH3T3 fibroblasts, which was inhibited by XCH, TGF- $\beta$ receptor inhibitor LY2157299, and shRNA targeting Hsp47. b The changes of a-SMA, HSP47, Timp-1, Col 1 and Col 3 protein level in $\mathrm{NIH} 3 \mathrm{~T} 3$ fibroblasts under specified treatments were evaluated by western blot. Abbreviations: SJ-A, S. japonicum egg antigens; LY, LY2157299. ${ }^{*} P<$ $0.05,{ }^{*} P<0.01$ compared to the control, $\# P<0.05$, \#\#P<0.01 compared to S. japonicum egg antigens

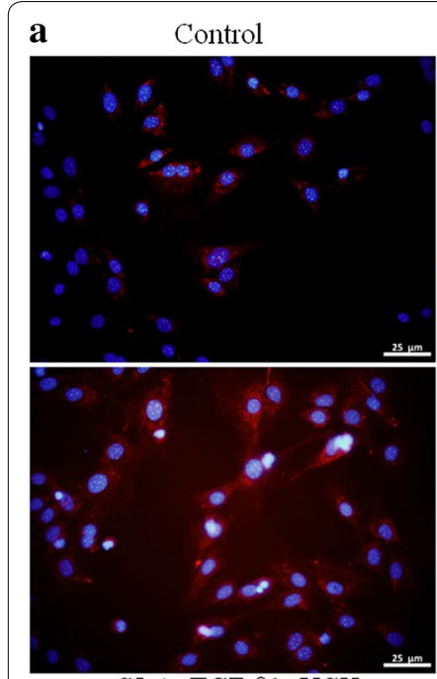

SJ-A+TGF- $\beta 1+\mathrm{XCH}$

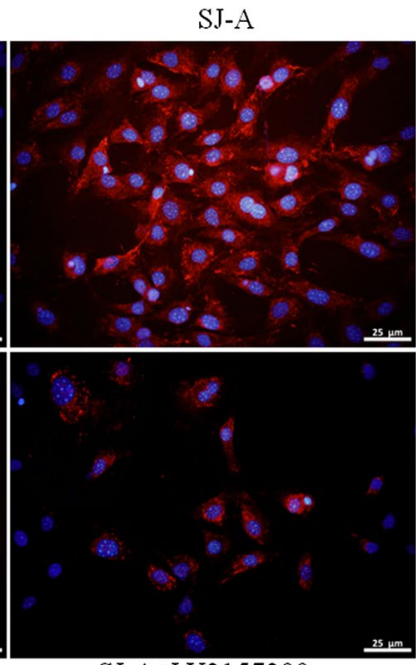

SJ-A+LY2157299
$\mathrm{SJ}-\mathrm{A}+\mathrm{XCH}$

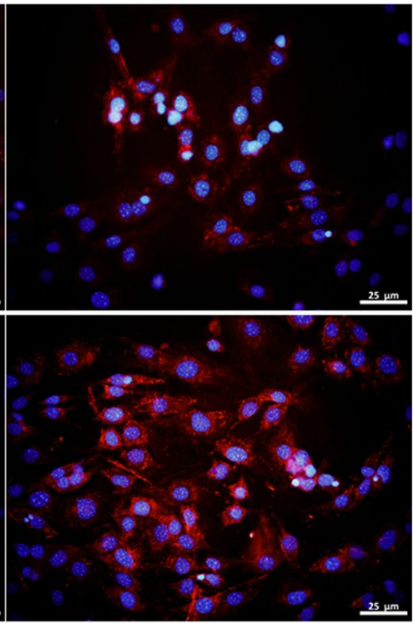

SJ-A+shRNANC
SJ-A+TGF- $\beta 1$

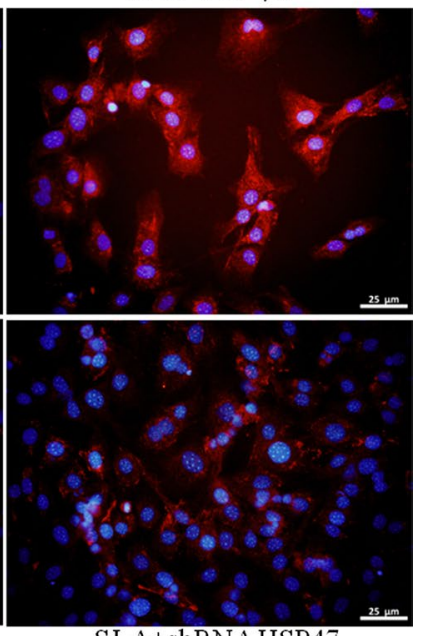

SJ-A+shRNA HSP47

b Control

$\mathrm{SJ}-\mathrm{A}+\mathrm{TGF}-\beta 1$

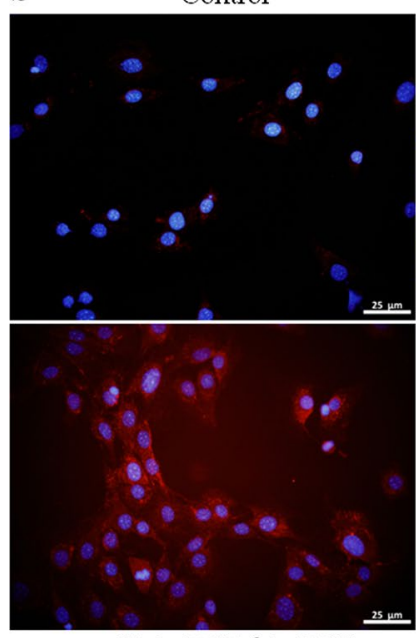

$\mathrm{SJ}-\mathrm{A}+\mathrm{TGF}-\beta 1+\mathrm{XCH}$ SJ-A

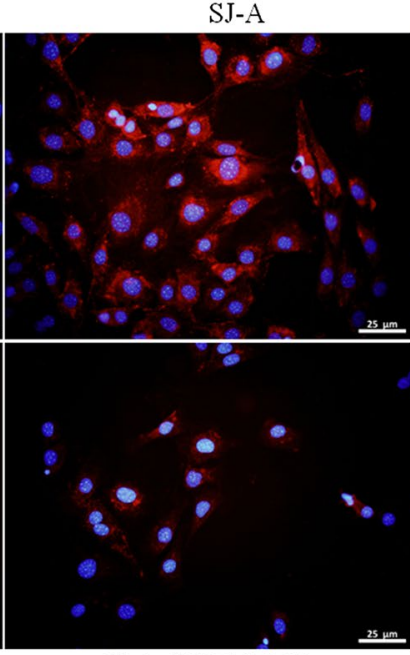

SJ-A+LY2157299
$\mathrm{SJ}-\mathrm{A}+\mathrm{XCH}$

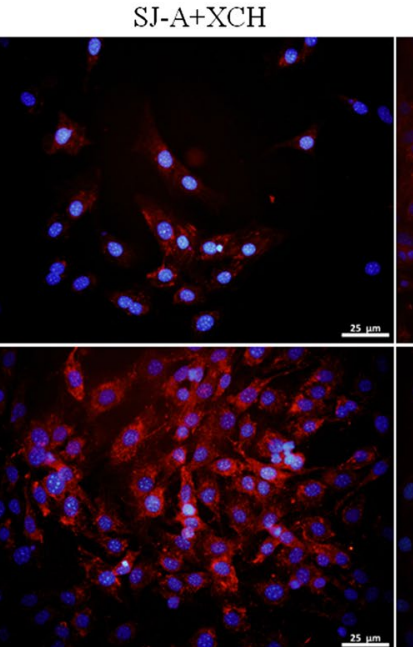

SJ-A+shRNANC

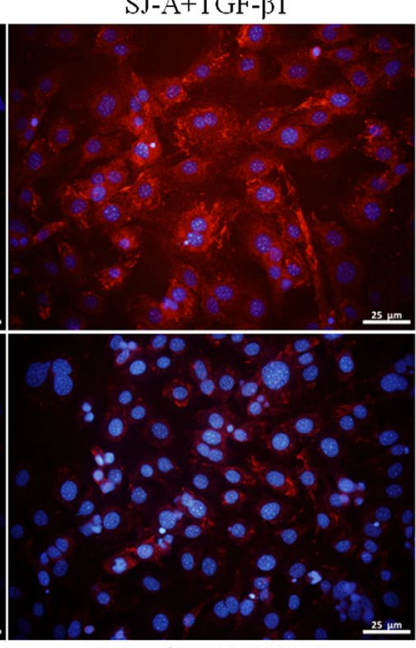

SJ-A+shRNA HSP47

Fig. 5 Xiaochaihu $(\mathrm{XCH})$ decoction reduced Schistosoma japonicum induced extracellular matrix deposition. The production and deposition of collagen 1 (a) and 3 (b) were assayed by immunofluorescence. Abbreviations: SJ-A, S. japonicum egg antigens; LY, LY2157299. Scale-bars: 25 m

\section{$\mathrm{XCH}$ inhibited the production of fibrogenetic cytokines} induced by S. japonicum egg antigens in NIH3T3 cells Schistosoma japonicum egg antigens strongly stimulated the excretion of TGF- $\beta 1$ (Fig. 6a), interleukin 13 (IL-13, Fig. 6b), and IL-17 (Fig. 6c) by NIH3T3 fibroblasts. Treatment with XCH, TGF- $\beta$ R1 antagonist LY2157299, and
shRNA-Hsp47 significantly reduced medium TGF- $\beta 1$ (ANOVA: $F_{(7,16)}=2520.5, P<0.0001$ ) (Fig. 6a), IL-13 (ANOVA: $F_{(7,16)}=774.8, P<0.0001$ ) (Fig. 6b), and IL-17 (ANOVA: $F_{(7,16)}=3481.2, P<0.0001$ ) (Fig. 6c) levels of NIH3T3 cells exposed to $S$. japonicum egg antigens. 

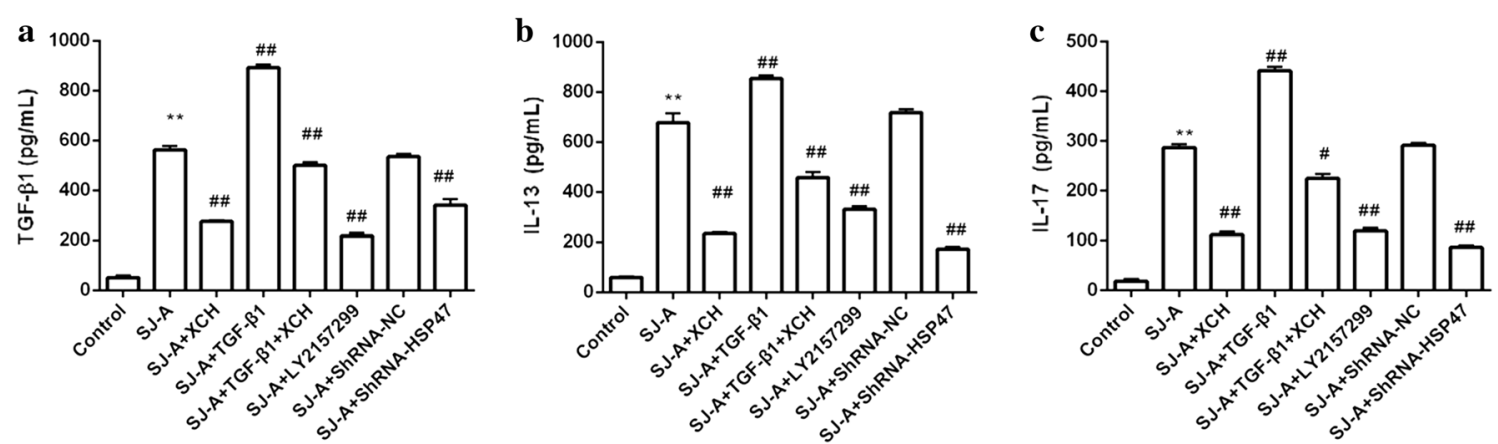

Fig. 6 Xiaochaihu $(\mathrm{XCH})$ decoction inhibited Schistosoma japonicum induced fibrogenic cytokine production by fibroblasts. NIH3T3 cells were treated with S. japonicum egg antigens in the presence or absence of Xiaochaihu decoction, LY2157299 and shRNA-HSP47. The medium levels of TGF- $\beta$ (a), IL-13 (b), and IL-17 (c) of NIH3T3 cells were measured by ELISA. Abbreviations: SJ-A, S. japonicum egg antigens. ${ }^{*} P<0.01$ compared to control, \#P<0.05 compared to infection, \#\#P<0.01 compared to infection
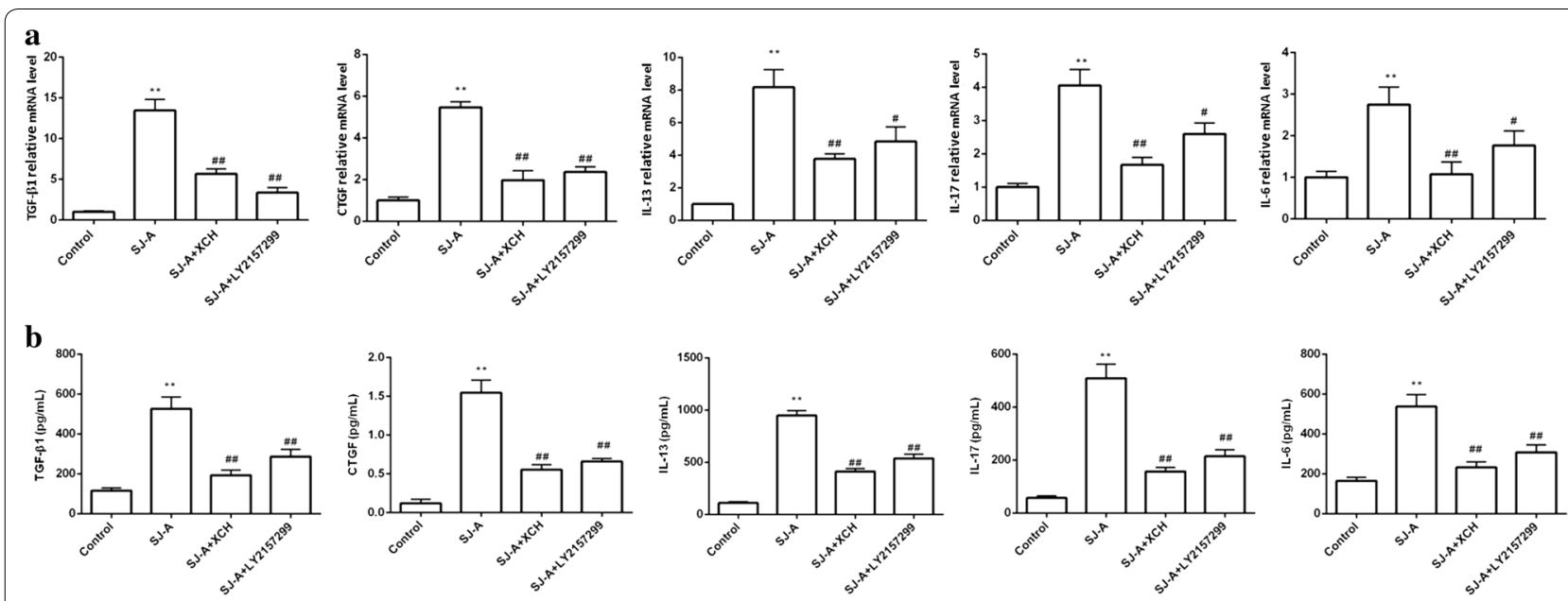

Fig. 7 Schistosoma japonicum induced activation of macrophages was inhibited by Xiaochaihu decoction (XCH). RAW264.7 cells were treated with S. japonicum egg antigens in the presence or absence of Xiaochaihu decoction and LY2157299. a The mRNA levels of TGF- $\beta 1$, CTGF, IL-13, IL-17 and IL-6 of Raw264.7 macrophages were analyzed by quantitative real-time PCR. b The levels of medium TGF- $\beta 1$, CTGF, IL-13, IL-17 and IL-6 of Raw264.7 macrophages were assessed by ELISA. Abbreviations: SJ-A, S. japonicum egg antigens. ${ }^{*} P<0.01$ compared to control, \#P<0.05 compared to infection, \#\#P<0.01 compared to infection

\section{XCH inhibited S. japonicum-induced activation of macrophages}

To investigate the effects of S. japonicum infection and Xiaochaihu decoction on the activation of macrophages, we treated Raw264.7 mouse macrophages with S. japonicum egg antigens in the presence or absence of $\mathrm{XCH}$ and LY2157299. Schistosoma japonicum egg antigens markedly increased the mRNA levels of TGF- $\beta 1$, CTGF, IL-13, IL-17 and IL-6, and the increases were significantly inhibited by either XCH or LY2157299 (ANOVA: TGF- $\beta 1, F_{(3}$, ${ }_{8}=1479.0, P<0.0001$, CTGF, $F_{(3,8)}=973.8, P<0.0001$; IL-13, $F_{(3,8)}=5157.7, P<0.0001$; IL-17, $F_{(3,8)}=33332.3$, $P<0.0001$; IL-6, $F_{(3,8)}=2839.5, P<0.0001$ ) (Fig. 7a). Medium levels of TGF- $\beta 1$, CTGF, IL-13, IL-17, and IL-6 of Raw264.7 macrophages were drastically increased by S. japonicum egg antigens (Fig. 7b). XCH and LY2157299 treatment reduced medium TGF- $\beta 1$, CTGF, IL-13, IL-17, and IL- 6 levels by $40-60 \%$ of $S$. japonicum egg antigenstreated Raw264.7 macrophages (ANOVA: TGF- $\beta 1, F_{(3}$, ${ }_{8)}=244.4, P<0.0001$; CTGF, $F_{(3,8)}=472.8, P<0.0001$; IL-13, $F_{(3,8)}=125.8, P=0.0004$; IL-17, $F_{(3,8)}=100.3, P=$ 0.0006; IL-6, $F_{(3,8)}=35.7, P=0.0039$ ) (Fig. 7b).

\section{Discussion}

The traditional Chinese medicine Xiaochaihu decoction showed a strong curative effect against $S$. japonicuminduced hepatic fibrosis via a Hsp47/TGF- $\beta 1$ axis. XCH reduced $S$. japonicum egg burden and fibrotic tissues in 


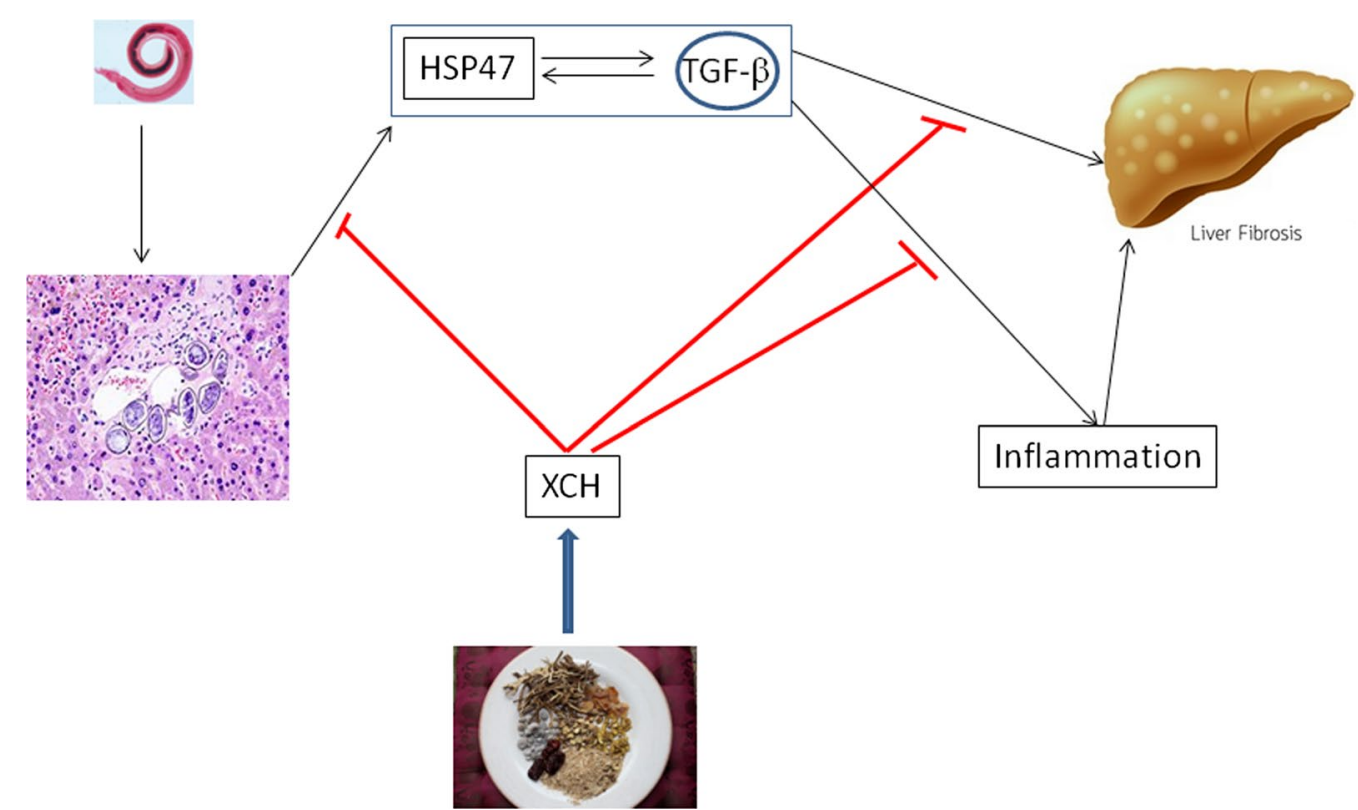

Fig. 8 A proposed model for explaining how XCH alleviates S. japonicum infection induced liver fibrosis. XCH inhibits S. japonicum infection, survival, and proliferation in mouse liver, depresses S. japonicum infection induced upregulation of HSP47 and TGF- $\beta$ production, and inhibits S. japonicum-caused inflammation, which in turn blocks the activation of hepatic stellate cells and ECM deposition

infected mouse livers, inhibited hepatic expression of fibrogenic genes TGF- $\beta 1$, Hsp47, $\alpha$-SMA, Col1A1 and Col3A1, upregulated by S. japonicum infection. Schistosoma japonicum caused changes in ALT, AST, ALP, ALB, GLOB, HA and PIIINP levels, which were considerably reversed by XCH. Schistosoma japonicum egg antigens induced the expression of fibrogenic genes Hsp47, TGF- $\beta 1$, Timp- $1, \alpha$-SMA, Col1A1 and Col3A1 in NIH3T3 mouse fibroblasts, which was inhibited by $\mathrm{XCH}$ and shRNA-Hsp47. Fibrogenic cytokines induced by $S$. japonicum egg antigens were inhibited by $\mathrm{XCH}$, LY2157299 and shRNA-Hsp47 in NIH3T3 fibroblasts and by XCH and LY2157299 in Raw264.7 macrophages.

Hepatic fibrosis is the most severe clinical consequence of $S$. japonicum infection $[5,28]$ so a great effort has been made to look for reagents protecting against liver fibrosis of infected individuals. Polyinosinic-polycytidylic acid (poly I:C) treatment reduced collagen deposition and stellate cell activation in S. japonicum-infected mouse liver accompanied by upregulation of Th1 cytokines interferons $\alpha, \beta$ and $\gamma$, TNF- $\alpha$, IL-10, and IL-12 and downregulation of Th2 cytokines IL-4 and IL-5 [29]. Boswellic acid-containing extracts of the oleogum resin from Boswellia serrata considerably reduced the size of liver granuloma and serum levels of ALT and AST but did not impact the egg burden in S. japonicum-infected mice [30]. Corilagin improved the health and reduced egg burden in S. japonicum-infected mice through blocking
TGF- $\beta$ signaling by upregulating SMAD7 and inhibiting the activation of SMAD1/2 and ERK1/2 [31]. Genistein protected against liver fibrosis caused by $S$. japonicum and decreased the extent of hepatic granuloma via inhibiting activation of NF- $\mathrm{kB}$ signaling pathway and led to downregulation of inflammatory cytokines MCP1, TNF $\alpha$, IL1 $\beta$, IL4, IL10 in infected mice [32]. These data indicate that some plant-derived compounds are effective in treating hepatic fibrosis caused by $S$. japonicum infection. The present study demonstrated the efficacy of Xiaochaihu decoction in reducing egg burden and liver fibrosis in $S$. japonicum infected mice.

Collagen-specific chaperon HSP47 has been shown to be involved in collagen-related diseases. HSP47 was found to be overexpressed in skin fibroblasts and peripheral blood mononuclear cells from scleroderma patients. Silencing HSP47 blocked TGF- $\beta$ induced collagen overproduction [33]. HSP47 was overexpressed in glioblastoma multiforme and promoted a stem-like property of primary glioma cells and the upregulation of extracellular matrix genes, which was inhibited by blocking the TGF- $\beta$ pathway [34]. Chronic graft-versus-host disease after allogeneic hematopoietic stem cell transplantation in mice showed massive skin fibrosis with elevated collagen deposition and F4/80+ macrophage infiltration, which was alleviated by HSP47 small interfering RNA delivered by vitamin A-coupled liposomes [16]. HSP47 was upregulated by $S$. japonicum infection and short hairpin 
(sh)RNA targeting Hsp47 markedly reduced collagen deposition in NIH3T3 cells and S. japonicum-infected mouse livers. The mouse survival rate was prolonged by shRNA-Hsp47 in a dose-dependent manner [35]. HSP47 was recently shown to modulate the activation of hepatic stellate cells by regulating the expression of endothelin receptor A and endothelin receptor B in S. japonicuminfected mice [36]. The current study demonstrated that $\mathrm{XCH}$ inhibited inflammatory responses and TGF- $\beta$ excretion of macrophages and the activation and ECM secretion of fibroblasts through inhabiting the expression of Hsp47 (Fig. 8).

\section{Conclusions}

The present data show that XCH potently inhibited the expression of Hsp47 of both S. japonicum-infected mouse liver and NIH3T3 cells, which led to downregulation of other fibrogenic genes IL-13, IL-17, TGF- $\beta 1$, Timp-1, $\alpha$-SMA, Col1A1 and Col3A1. The remarkably decreased levels of fibrogenic cytokines, signaling proteins and extracellular matrix-related proteins resulted in a significant relieve of hepatic fibrosis induced by $S$. japonicum. These results indicate that Xiaochaihu decoction might be an effective therapeutic option for liver fibrosis caused by $S$. japonicum infection.

\section{Supplementary information}

\section{Supplementary information accompanies this paper at https://doi.} org/10.1186/s13071-020-04121-2.

Additional file 1: Table S1. Sequence information for primers used in the study.

\section{Abbreviations}

XCH: Xiaochaihu decoction; PCR: polymerase chain reaction; ELISA: enzymelinked immunosorbent assay; ALT: alanine aminotransferase; AST: aspartate aminotransferase; ALP: alkaline phosphatase; HA: hyaluronic acid; PIIINP: amino-terminal pro-peptide of type III pro-collagen; ALB: albumin; GLOB: globulin; TGF- $\beta 1$ : transforming growth factor beta 1; Hsp47: heat shock protein 47; a-SMA: alpha smooth muscle actin; Col1A1: collagen type I alpha 1 chain; Col3A1: collagen type III alpha 1 chain; CTGF: connective tissue growth factor; IL: interleukin; dpc: days post-coitus; KO: knockout; PVDF: polyvinylidene fluoride; RT-qPCR: real-time quantitative polymerase chain reaction.

\section{Acknowledgements}

Dr. Wu Qijun from Wuxi hospital affiliated of Nanjing University of Chinese Medicine provide Xiaochaihu decoction. Professor Yang Junqi, Dr. Song Lijun, Professor Zhou Yonghua, Dr. Zhang Ying and Dr. Hou Ru provide some technical support and advice for the research.

\section{Authors' contributions}

YH and HY conceived the project and designed the study. JL, YX, CX, DT and $\mathrm{NH}$ performed the experiments. $\mathrm{YH}$ analyzed the data and $\mathrm{YH}$ and $\mathrm{HY}$ wrote the manuscript. All authors read and approved the final manuscript.

\section{Funding}

This study was supported by the Natural Science Foundation of China (grant No. 81673673); Project of Public Health Research Center of Jiangnan University (grant No. JUPH201811); funding of Wuxi Science and Technology development (WX0302B010504180001 PB); the Project of Invigorating Health Care through Science, Technology and Education No. ZDXKA2016016, the Jiangsu Provincial Department of Science and Technology No. BM2018020; the Jiangsu Provincial Department of Science and Technology (BE2016631 and BM2015024) and the Jiangsu Health International Exchange Program awarded to $\mathrm{YH}$. The funders had no role in study design, data collection, management, analysis and interpretation, decision to publish, or in the preparation, review or approval of this manuscript.

\section{Availability of data and materials}

Data supporting the conclusions of this article are included within the article and its additional file.

\section{Ethics approval and consent to participate}

All experiments described here were fully complied with the current laws of the country in which the experiments were performed (P.R. China) and approved by the institutional animal care and usage committee of Jiangsu Institute of Parasitic Diseases (JIPD_2018001036).

\section{Consent for publication}

Not applicable.

\section{Competing interest}

The authors declare that they have no competing interests.

\section{Author details}

${ }^{1}$ National Health Commission Key Laboratory of Parasitic Disease Control and Prevention, Jiangsu Provincial Key Laboratory on Parasite and Vector Control Technology, Jiangsu Institute of Parasitic Diseases, 117 Meiyuan Yangxiang, Wuxi 214064, Jiangsu, China. ${ }^{2}$ Public Health Research Center, Jiangnan University, Wuxi 214122, Jiangsu Province, China. ${ }^{3}$ Center for Global Health, School of Public Health, Nanjing Medical University, Nanjing 211166, China.

Received: 7 September 2019 Accepted: 6 May 2020

Published online: 14 May 2020

References

1. McManus DP, Dunne DW, Sacko M, Utzinger J, Vennervald BJ, Zhou XN. Schistosomiasis. Nat Rev Dis Primers. 2018:4:13.

2. Kapoor S. Katayama syndrome in patients with schistosomiasis. Asian Pac J Trop Biomed. 2014:4:244.

3. Olveda DU, Olveda RM, McManus DP, Cai P, Chau TN, Lam AK, et al. The chronic enteropathogenic disease schistosomiasis. Int J Infect Dis. 2014;28:193-203.

4. Dang H, Xu J, Li SZ, Cao ZG, Huang YX, Wu CG, et al. Monitoring the transmission of Schistosoma japonicum in potential risk regions of China, 2008-2012. Int J Environ Res Public Health. 2014;11:2278-87.

5. Chen MG. Assessment of morbidity due to Schistosoma japonicum infection in China. Infect Dis Poverty. 2014:3:6.

6. Chen D, Luo X, Xie H, Gao Z, Fang H, Huang J. Characteristics of IL-17 induction by Schistosoma japonicum infection in C57BL/6 mouse liver. Immunology. 2013:139:523-32.

7. Chuah C, Jones MK, Burke ML, McManus DP, Gobert GN. Cellular and chemokine-mediated regulation in schistosome-induced hepatic pathology. Trends Parasitol. 2014;30:141-50.

8. Koyama Y, Brenner DA. Liver inflammation and fibrosis. J Clin Invest. 2017;127:55-64.

9. Cao S, Zhu C, Feng J, Zhu L, Yin J, Xu Y, et al. Helicobacter hepaticus infection induces chronic hepatitis and fibrosis in male BALB/C mice via the activation of NF-KB, Stat3, and MAPK signaling pathways. Helicobacter. 2019:27:e12677.

10. Kamdem SD, Moyou-Somo R, Brombacher F, Nono JK. Host regulators of liver fibrosis during human schistosomiasis. Front Immunol. 2018;9:2781.

11. Luo W, Xu Q, Wang Q, Wu H, Hua J. Effect of modulation of PPAR- $\gamma$ activity on Kupffer cells M1/M2 polarization in the development of non-alcoholic fatty liver disease. Sci Rep. 2017:7:44612.

12. Ishida Y, Nagata K. Hsp47 as a collagen-specific molecular chaperone Methods Enzymol. 2011:499:167-82. 
13. Nagata K. Hsp47: a collagen-specific molecular chaperone. Trends Biochem Sci. 1996;21:22-6.

14. Nagai N, Hosokawa M, Itohara S, Adachi E, Matsushita T, Hosokawa $\mathrm{N}$, et al. Embryonic lethality of molecular chaperone hsp47 knockout mice is associated with defects in collagen biosynthesis. J Cell Biol. 2000;150:1499-506.

15. Masago Y, Hosoya A, Kawasaki K, Kawano S, Nasu A, Toguchida J, et al. The molecular chaperone Hsp47 is essential for cartilage and endochondral bone formation. J Cell Sci. 2012;125:1118-28.

16. Ito S, Nagata K. Biology of Hsp47 (Serpin H1), a collagen-specific molecular chaperone. Semin Cell Dev Biol. 2017;62:142-51.

17. Khan ES, Sankaran S, Paez JI, Muth C, Han MKL, Del Campo A. Photoactivatable Hsp47: a tool to regulate collagen secretion and assembly. Adv Sci. 2019;6:1801982.

18. Yamakawa T, Ohigashi H, Hashimoto D, Hayase E, Takahashi S, Miyazaki $M$, et al. Vitamin A-coupled liposomes containing siRNA against HSP47 ameliorate skin fibrosis in chronic graft-versus-host disease. Blood. 2018;131:1476-85.

19. Otsuka M, Shiratori M, Chiba H, Kuronuma K, Sato Y, Niitsu Y, et al. Treatment of pulmonary fibrosis with siRNA against a collagen-specific chaperone HSP47 in vitamin A-coupled liposomes. Exp Lung Res. 2017:43:271-82.

20. Wu YQ, Gou YQ, Han J, Bi YY, Feng SL, Hu FD, et al. Evaluation preparation technology of Xiaochaihu granules using fingerprint-peak pattern matching. J Pharm Anal. 2011;1:119-24.

21. Ohta Y, Nishida K, Sasaki E, Kongo M, Hayashi T, Nagata M, et al. Comparative study of oral and parenteral administration of sho-saiko-to (xiao-chaihu-tang) extract on D-galactosamine-induced liver injury in rats. Am J Chin Med. 1997;25:333-42.

22. Nose M, Terawaki K, Iwahashi N, Oguri K, Ogihara Y. Comparative study of the high molecular mass fraction and low molecular mass fraction of Sho-saiko-to in a murine immunologically induced liver injury model. Biol Pharm Bull. 2002;25:64-7.

23. Bachem MG, Meyer D, Schäfer W, Riess U, Melchior R, Sell KM, et al. The response of rat liver perisinusoidal lipocytes to polypeptide growth regulator changes with their transdifferentiation into myofibroblast-like cells in culture. J Hepatol. 1993;18:40-52.

24. Ono M, Miyamura M, Kyotani S, Saibara T, Ohnishi S, Nishioka Y. Effect of Sho-saiko-to extract on HGF and TGF-beta levels of intraorgans in liverinjured rats after partial hepatectomy. J Pharm Pharmacol. 2000;52:111-8.

25. Zhang SK, Cui NQ, Zhuo YZ, Li DH, Liu JH. Modified Xiaochaihu Decoction prevents the progression of chronic pancreatitis in rats possibly by inhibiting transforming growth factor- $\beta 1 / \mathrm{Sma-}$ and mad-related proteins signaling pathway. Chin J Integr Med. 2013;19:935-9.

26. Huang Y, Cai B, Xu M, Qiu Z, Tao Y, Zhang Y, et al. Gene silencing of Toll-like receptor 2 inhibits proliferation of human liver cancer cells and secretion of inflammatory cytokines. PLoS One. 2012;7:e38890.

27. Huang Y, Li W, Liu K, Xiong C, Cao P, Tao J. New detection method in experimental mice for schistosomiasis: ClinProTool and matrix-assisted laser desorption/ionization time-of-flight mass spectrometry. Parasitol Res. 2016:115:4173-81.

28. Huang $Y, X u Y$, Huang $Y$, Sun F, Tian H, Hu N, et al. Identification of newly developed advanced schistosomiasis with MALDI-TOF mass spectrometry and ClinProTools analysis. Parasite. 2019;26:33.

29. Hou X, Yu F, Man S, Huang D, Zhang Y, Liu M, et al. Polyinosinic-polycytidylic acid attenuates hepatic fibrosis in C57BL/6 mice with Schistosoma japonicum infection. Acta Trop. 2012;121:99-104.

30. Liu M, Chen P, Büchele B, Dong S, Huang D, Ren C, et al. A boswellic acid-containing extract attenuates hepatic granuloma in C57BL/6 mice infected with Schistosoma japonicum. Parasitol Res. 2013;112:1105-11.

31. Yang F, Wang Y, Xue J, Ma Q, Zhang J, Chen YF, et al. Effect of Corilagin on the miR-21/smad7/ERK signaling pathway in a schistosomiasis-induced hepatic fibrosis mouse model. Parasitol Int. 2016;65:308-15.

32. Wan C, Jin F, Du Y, Yang K, Yao L, Mei Z, et al. Genistein improves schistosomiasis liver granuloma and fibrosis via dampening NF-kB signaling in mice. Parasitol Res. 2017;116:1165-74.

33. Chu H, Wu T, Wu W, Tu W, Jiang S, Chen S, et al. Involvement of collagenbinding heat shock protein 47 in scleroderma-associated fibrosis. Protein Cell. 2015;6:589-98.

34. Jiang X, Zhou T, Wang Z, Qi B, Xia H. HSP47 Promotes glioblastoma stemlike cell survival by modulating tumor microenvironment extracellular matrix through TGF- $\beta$ pathway. ACS Chem Neurosci. 2017;8:128-34.

35. Huang JQ, Tao R, Li L, Ma K, Xu L, Ai G, Fan XX, Jiao YT, Ning Q. Involvement of heat shock protein 47 in Schistosoma japonicum-induced hepatic fibrosis in mice. Int J Parasitol. 2014:44:23-35.

36. Zhao Y, Dang Z, Xu S, Chong S. Heat shock protein 47 effects on hepatic stellate cell-associated receptors in hepatic fibrosis of Schistosoma japonicum-infected mice. Biol Chem. 2017;398:1357-66.

\section{Publisher's Note}

Springer Nature remains neutral with regard to jurisdictional claims in published maps and institutional affiliations.
Ready to submit your research? Choose BMC and benefit from:

- fast, convenient online submission

- thorough peer review by experienced researchers in your field

- rapid publication on acceptance

- support for research data, including large and complex data types

- gold Open Access which fosters wider collaboration and increased citations

- maximum visibility for your research: over 100M website views per year

At BMC, research is always in progress.

Learn more biomedcentral.com/submissions 